\title{
Mechanical Properties and Gradient Effects of Carbon Nanotube as Polymer Nanocomposites
}

\author{
N. Abu-Hamdeh \\ Mechanical Engineering Department, King Abdulaziz \\ University \\ Jeddah, Saudi Arabia
}

\author{
K. Alnefaie \\ Mechanical Engineering Department, King Abdulaziz \\ University \\ Jeddah, Saudi Arabia
}

\begin{abstract}
Carbon nanotubes (CNTs) were fabricated using low pressure chemical vapor deposition (LPCVD) and then embedded in epoxy polymer at several weight ratios; $0,0.75,1.5$, and 3 (wt \%) for tensile testing and Young's modulus determination using intron machine. Tensile strength and Young's modulus of the epoxy resin were increased with the addition of CNTs to a certain extend and then decreased with the increase in weight fraction of carbon nanotubes. The best properties occurred at 1.5 (wt \%) of CNTs. Scanning electron microscopy was used to reveal the dispersion status of carbon nanotubes in the nanocomposites.
\end{abstract}

Keywords- carbon nanotubes; polymer nanocomposites; epoxy polymer; mechanical properties

\section{INTRODUCTION}

Polymer is a long molecule consisting of many small units (monomers) connected end-to-end. Polymers have low stiffness and strength compared to metals. These properties can be improved by adding reinforcements such as fibers, tubes or particles. The reinforced polymeric material is known as composite material with non-isotropic physical properties but orthotropic. On the other hand, a nanocomposite material is defined as a multiphase solid material with one of the phases consists of at least one dimension of less than $100 \mathrm{~nm}$, or constructions having nano-scale recurrence intervals between the different phases that form the material [1].

Materials comprised from nanometer-size particles have been found to produce exceptional mechanical properties compared to their micron-size equivalents. At room temperature, Mayo et al. [2] obtained strain-rate sensitivity measurements that indicate ductile behavior of nano-phase ceramics. Guermazi [3] reported substantial deformation of dense nano-phase $\mathrm{TiO}_{2}$ without fracture or cracking at elevated temperature. Similarly, Jain and Christman [4] reported similar results in their experiments on a crusted nano-phase inter metallic composite. Chen et al. [5] compared the nano and micro sizes of $\mathrm{TiO}_{2}$ filler in an epoxy resin. They found that nano composite exhibited a remarkable increase in the modulus and the strain to failure as compared to pure epoxy and micro composite.

The production of nanotubes in large quantities can be done using different techniques. These include chemical vapor deposition (CVD), arc discharge, laser ablation, and high pressure carbon monoxide. The chemical vapor deposition (CVD) is the most promising for industrial-scale deposition. It took until 1993 [6] for nanotubes to be actually produced by this technique even though the CVD of carbon was initially stated by [7] in 1959. Recently, $18 \mathrm{~cm}$ arrays length of aligned carbon nanotube was reached by Wang et al. [8].

Research studies in the literature presented strong evidence that carbon nanotubes have remarkable mechanical properties. Due to their exceptional thermomechanical, chemical and optoelectronic properties, carbon nanotubes (CNTs) are becoming increasingly promising materials for modern technology applications (nanocomposites, nanodevices, nanoelectronics, nanomedicine). In spite of this, the explicit determination of the mechanical properties of these nanotubes is quite a challenging assignment due to the technical obstacles engaged in handling these nanotubes. Experimental measurements and theoretical estimates on these materials have shown that the elastic modulus of these nanotubes is between 0.5 and $1 \mathrm{TPa}$ and tensile strength in the range of 50$200 \mathrm{GPa}$, which makes them perfect candidates for composites reinforcement [9]. The superior strength and unusual deformability of CNTs have been a subject of a number of recent studies, specifically the dispersing of carbon nanotubes in low strength polymers to improve their mechanical properties [10]. Although the reported measurements of modulus values for carbon nanotubes were high, polymer nanocomposites have not exhibited the anticipated scales of stiffness improvement because of few crucial matters mainly associated to morphology and processing [10]. For example, Cebeci et al. [11] investigated the elastic modulus of a composite formed from aligned multi-walled carbon nanotubes having $0.5 \mathrm{TPa}$ modulus embedded in an aerospace grade structural epoxy resin. The measured composite modulus was $8.8 \mathrm{GPa}$ while the expected value was $100 \mathrm{GPa}$. Moreover, they found that the elastic modulus of the composite increased with increasing the volume fraction of the embedded multi-walled nanotubes.

This research article exhibits the fabrication of carbon nanotubes (CNTs) using low pressure chemical vapor deposition (LPCVD) to be embedded in epoxy polymer at several weight ratios for tensile testing and Young's modulus determination.

\section{EXPERIMENTAL AND METHODS}

\section{A. Materials}

Low pressure chemical vapor deposition (LPCVD) method was used in this study to grow the carbon nanotubes. The 
average diameter and length of the tubes were found to be 20 and $1000 \mathrm{~nm}$, respectively. The hardener (curing agent) was cobalt naphthenate, approximately $53 \%$ in mineral spirits $(6 \%$ Co) and MEKP (Alfa Aesar® ${ }^{\circledR}$, Johnson Matthey Management GmbH \& Co Karlsruhe Germany). Other materials used were: ethanol as solvent to dilute the epoxy resin, mold release agent (Ease Release® 200), Fosroc Solvent 102.

\section{B. Preparation Method}

A substrate of silicon wafer was coated with iron nano particles as a catalyst using LPCVD machine manufactured by Syskey technology Ltd. Ferrocene was used as a catalyst while acetylene $\left(\mathrm{C}_{2} \mathrm{H}_{2}\right)$ was used as a carbon precursor along with nitrogen $\left(\mathrm{N}_{2}\right)$ as a process gas. Carbon nanotube at $0,0.75,1.5$, and 3 (wt \%) of epoxy resin samples were prepared. First, carbon nanotubes were dispersed in ethanol and mixed mechanically for 10 minutes at 2,000 rpm. Then, epoxy resin was added to this solution. Next, in order to obtain homogeneous dispersion, the mixture was sonicated by an ultrasonicator machine, BRANSON 2510, for 12 hours. For the removal of the ethanol, the mixture was then placed in a low-power ultrasonic bath (BRANSON 2510) for another 12 hours. The residual ethanol was removed by evaporation in vacuum. After that, the hardener was added to the combination and mechanically mixed for 20 minutes. Finally, the mixture was molded and cured at room temperature.

\section{Characterization Methods}

1) Micro Raman spectroscopy. Micro Raman spectrometer was used to determine if the tested samples were taking more of a graphite shape than an amorphous or vice versa. Raman spectrum was measured using DXR Raman microscope from Thermo Scientific ${ }^{\circledR}$ using $532 \mathrm{~nm}$ laser as excitation source at power of $8 \mathrm{~mW}$. Spectrum was measured in the $50-3600 \mathrm{~cm}^{-1}$ zone. The spectral resolution is $3 \mathrm{~cm}^{-1}$. The result indicated that the structure of the carbon nanotubes taking more of graphite shape.

2) Scanning electron microscopy. The morphologies of the resulted carbon nanotubes were analyzed by scanning electron microscopy. Scanning electron microscopy (SEM) images were captured for the fabricated nanotubes. The microscopic morphology was examined by the FEI $\mathbb{R}$ Magellan ${ }^{\mathrm{TM}} 400 \mathrm{~L}$ system. Transmission electron microscopy (TEM) image of CNTs at high resolution was also obtained.

3) Mechanical analysis. To evaluate the effects of adding CNTs on the tensile strength of the composite samples, tensile tests were conducted on the cured samples according to ASTM D638 using the universal testing machine, INSTRON ${ }^{\circledR} 3369$, at a load of $50 \mathrm{kN}$ and a rate of $5 \mathrm{~mm} / \mathrm{min}$.

\section{RESULTS AND DISCUSSION}

\section{A. SEM and Tem Results}

A set of techniques can be used to achieve characterization of carbon nanostructures. Electron microscopy techniques (SEM and TEM) are among of those most frequently used methods. Figure 1 depicts a SEM image of carbon nanotubes at low and high magnifications at the 3 (wt \%) of CNTs. The figure shows the yielded nanotubes shuffled in a noodle-like manner with a considerable yield of $\mathrm{Y}$ carbon nanotube junctions. As seen in the figure, there is a significant aggregation of carbon nanotubes in the CNT/epoxy nanocomposites at 3 (wt \%) of nanotubes. This would affect the strength of nanocomposites since these aggregates form stress concentration regions. The inner heart of these CNTs was repeatedly constantly filled with the catalyst material and indicated its role in facilitating of filling of tubes.

Figure 2 shows a high resolution transmission electron microscopy (HR TEM) image of carbon nanotubes. The (HR TEM) technique is effectively essential in assessing the characteristics of CNTs as this method can see samples at greater enlargements. As shown in Figure 2, the CNTs were distributed uniformly in size. This distribution was affected by the diameter distribution of the particles used. The figure also shows the formation of buds and multiple Y-junction carbon nanotubes.

\section{B. Mechanical Properties}

The impact of nanoparticles dispersion in epoxy resin on the mechanical properties of nanocomposites was the main objective of this research study. Figure 3 shows the tensile strength results of CNT/epoxy composites. Based on the results achieved, about 5.8\% enhancement on tensile strength was obtained by adding 0.75 (wt \%) of CNT to epoxy resin. Moreover, increasing the amount of CNT content increased the tensile strength which reached a peak improvement of $11.5 \%$ at 1.5 (wt \%) of CNT content and then decreased at 3 (wt \%) of CNT content. Figure 4 shows the Young's modulus of the resulting nanocomposites. Similar to the results of tensile strength, the optimum amount of Young's modulus was attained at 1.5 (wt \%) of CNT content. At 3 (wt \%) of CNT, both tensile strength and Young's modulus were decreased. Random orientation and aggregation of the carbon nanotubes inside the nanocomposites at higher weight fraction of CNTs might be the reason for such a conduct. Another possible reason that might be responsible for such an act is the dispersion status of CNTs. Attaining a homogenous dispersion is a challenging task because of their intense trend to aggregate.

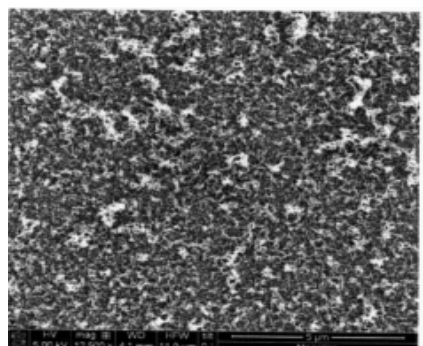

(A)

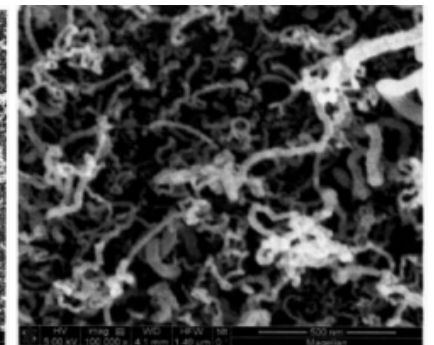

(B)
FIGURE I. (A) EXAMPLE OF SEM IMAGE OF THE CNTS AT LOW MAGNIFICATION, (B) EXAMPLE OF SEM IMAGE OF THE CNTS AT HIGH MAGNIFICATION (CASE 3 WT \%). 


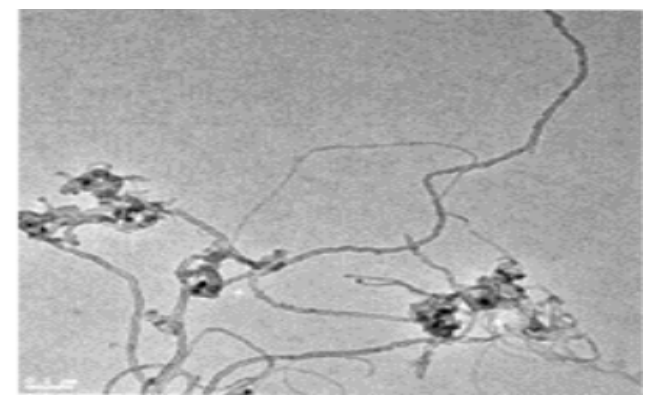

FIGURE II. HR TEM IMAGE OF THE CNTS AT 0.1 MICRO METERS.

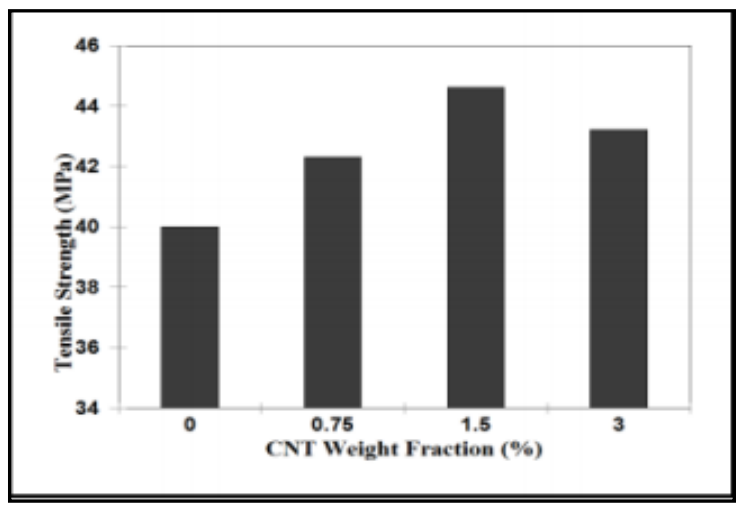

FIGURE III. TENSILE STRENGTH OF CNT/EPOXY NANOCOMPOSITES IN VARIOUS CNT WEIGHT FRACTION

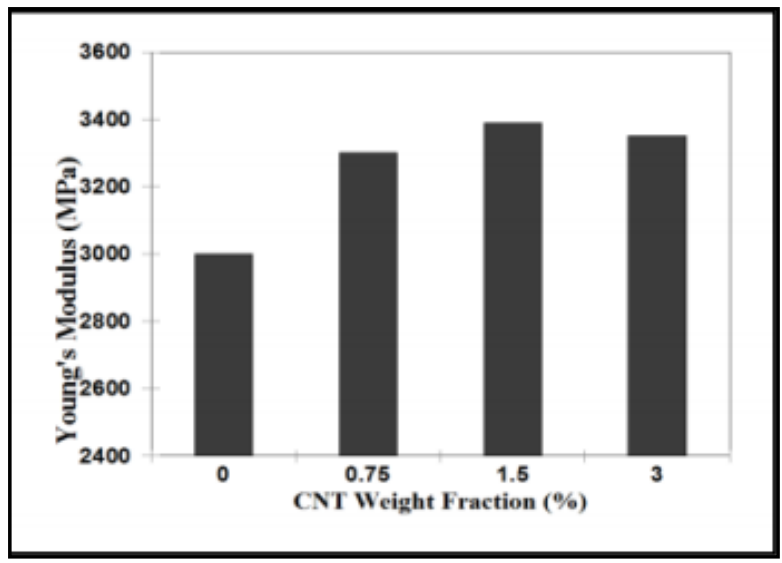

FIGURE IV. YOUNG'S MODULUS OF CNT/EPOXY NANOCOMPOSITES.

\section{ACKNOWLEDGMENT}

The project was funded by the Deanship of Scientific Research (DSR), King Abdulaziz University, Jeddah, under grant no. (161/135/1434). The authors, therefore, acknowledge with thanks DSR technical and financial support.

\section{REFERENCES}

[1] Ajayan, P. M., Schadler, L. S., Braun, P. V., Nanocomposite science and technology. Wiley-VCH Verlag GmbH \& Co. KGaA, Germany, 2004.

[2] Mayo, M. J., Siegel, R. W., Naryanasamy, A., Nix, W. D., Mechanical properties of nanophase $\mathrm{TiO} 2$ as determined by nanoindentation, J. Mater. Res., 5, 1073-1082, 1990.
[3] Guermazi, M., Hofler, H. J., Hahn, H., Averback, R. S., Temperaturedependence of the hardness of nanocrystalline titanium-dioxide,” J. Am. Ceram. Soc., 74, 2672-2680, 1991.

[4] Jain, M. Christman, T., Synthesis, processing, and bulk deformation of bulk nanophase Fe-28Al-2Cr intermetallic, Acta. Metall. Mater., 42, 1901-1911, 1994.

[5] Chen, Q., Schadler, L. S,. Siegel, R. W., Irvin, G. C., Mendel, J., Enhanced scratch resistance of nanocomposite gelatin films, in Materials Research Society Symposium Proceedings-Symposium T-Polymer Nanocomposites, S. Nutt, R.Vaia, W. Rodgers, G. L. Hagnauer, and G. W. Beall, eds. Curran Associates, Inc., California-USA, 2002.

[6] Jose-Yacaman, M., Miki-Yoshida, M., Rendon, L., Santiesteban, J. G., Catalytic growth of carbon microtubes with fullerene structure, Appl. Phys. Lett., 62, 657- 659, 1993.

[7] Walker, P. L., Rakszawski, J. F., Imperial, G. R., Carbon formation from carbon monoxide-hydrogen mixtures over iron catalysts. I. Properties of carbon formed, J. Phys. Chem., 63, 133-140, 1959.

[8] Wang, X., Li, Q., Xie, J., Jin, Z., Wang, J., Li, Y., Jiang, K., Fan, S., Fabrication of ultralong and electrically uniform single-walled carbon nanotubes on clean substrates, Nano. Lett., 9, 3137-3141, 2009.

[9] Treacy, M. M. T., Ebbesen, T. W., Gibson, J. M., Exceptionally high Young's modulus observed for individual carbon nanotubes, Nature, 381 678-680, 1996.

[10] Wong, E. W., Sheehan, P. E., Lieber, C. M., Nanobeam mechanics: elasticity, strength, and toughness of nanorods and nanotubes, Science, 277, 1971-1975, 1997.

[11] Cebeci, H., Villoria, R. G., Hart, A. J., Wardle, B. L., Multifunctional properties of high volume fraction aligned carbon nanotube polymer composites with controlled morphology, Compos. Sci. Technol., 69, 2649-2656, 2009. 